\title{
MARTUCCELLI, DANILO (2015). LIMA Y SUS ARENAS. PODERES SOCIALES Y JERARQUÍAS CULTURALES. LIMA: CAUCES EDITORES
}

\author{
Nekson PIMENTEL SÁNCHEZ \\ Universidad Nacional Mayor de San Marcos \\ neksonunmsm@hotmail.com
}

D anilo Martuccelli es sociólogo de origen peruano, realizó sus estudios de grado en Argentina y desde la década del ochenta vive en Francia. Es profesor de sociología de la Facultad de Ciencias Humanas y Sociales de la Universidad París-Descartes; dedicado al estudio de la teoría social, sociología política y sociología del individuo'. Sus trabajos están orientados a explicar los cambios contemporáneos y buscan entrelazar las experiencias individuales con las estructuras sociales.

Este ensayo, basado en fuentes

Danilo Martuccelli

Lima y sus arenas Poderes sociales y jerarquías culturales

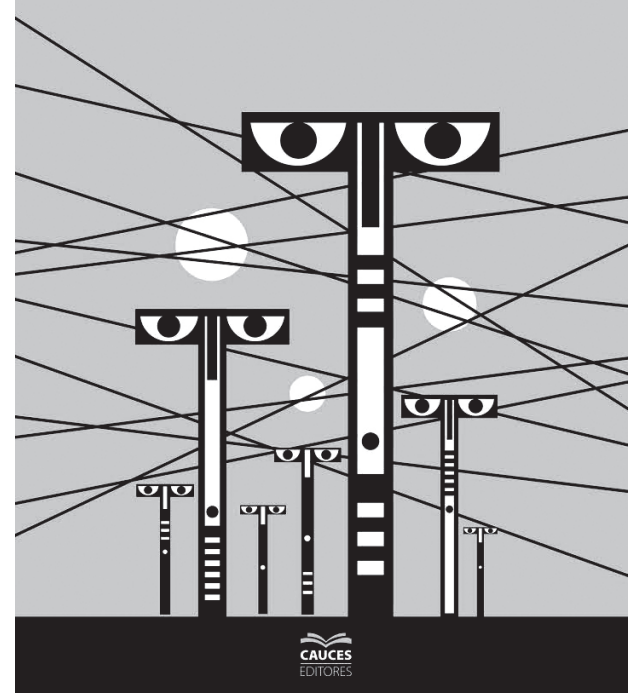
secundarias antes que en información recopilada mediante trabajo de campo, es una interpretación sociológica e histórica de la sociedad limeña de las últimas décadas. Sintetiza las raíces del conjunto dispar de transformaciones estructurales que ha terminado produciendo tres grandes procesos de cambios actualmente en curso en la sociedad limeña: proyecto reglamentador, revolución en la sociabilidad de raigambre popular que progresivamente se volvió mayoritaria $y_{\text {, }}$ finalmente, la constitución de lo que el autor denomina como un individualismo metonímico.

Estos procesos transformaron a Lima en una ciudad moderna. La transformación de Lima sería el resultado de cambios estructurales paralelos, a veces simultáneos entre sí, pero por lo general diferentes en sus orientaciones. Los elementos asociados al gran cambio incluyen la expansión urbana, la migración andina y provinciana, el colapso de los partidos políticos,

1 Véase, por ejemplo, Martuccelli, Danilo (2007). Cambio de rumbo. La sociedad a escala del individuo. Santiago: LOM Ediciones. 
la economía informal, la expansión de la escolaridad, la violencia política o delictiva y la reorientación macroeconómica neoliberal.

Lima y sus arenas. Poderes sociales y jerarquías culturales, con una mirada de aspectos específicos y de conjunto, es una de las interpretaciones novedosas sobre las transformaciones y experiencias de Lima de las últimas décadas. Mediante una estructura en dos partes, cada una con tres capítulos, además de las conclusiones, analiza y describe la importancia de las relaciones interpersonales, la asociación de la reglamentación con la informalidad, jerarquías culturales, ciudadanía, procesos de horizontalización, poderes sociales, así como las sinuosas y débiles conexiones entre política, cultura y economía en los cambios experimentados por la sociedad limeña.

En el primer capítulo, el autor realiza una lectura genealógica sobre los avatares del pueblo. Parte de la premisa que la consolidación de las individualidades limeñas contemporáneas es inseparable de la dificultad que tuvo la sociedad peruana, durante todo el siglo XX, para articular una experiencia nacional-popular como un modelo de identidad popular, cuya crisis permitió la aparición de una sociabilidad limeña.

El modelo nacional-popular, según el autor, persiguió una construcción política del pueblo ${ }^{2}$ como nación, y tuvo tres grandes expresiones truncas que abrieron el camino a una constitución más autónoma y cultural-social que política de lo popular: el avatar del APRA y de Haya de la Torre (desde 1930 hasta fines de los sesenta), el gobierno militar de Velasco Alvarado (1968-1975) y la primera gestión de Alan García (1985-1990).

Respecto a la primera -el APRA y de Haya de la Torre- es enfático señalar que la penetración del aprismo en las clases trabajadoras y, en general, en Lima fue débil. El APRA fue esencialmente el partido de las clases medias provincianas, más tarde urbanas, y de ciertos sectores "oligárquicos" amenazados por la penetración del capital extranjero. La interpretación de Haya de la Torre era la de una sociedad dualista en la cual se imponía la necesidad de una burguesía nacional, la que reconocía un rol importante al Estado y que se articuló en torno al antiimperialismo, el indigenismo y el relativismo histórico. El capitalismo en el Perú, para Haya, no era el fruto de un proceso interno de maduración sino el resultado de una importación que se injertó en sectores precapitalistas. De ahí, parafraseando a Lenin, Haya consideró al imperialismo como la primera etapa del capitalismo en Indoamérica, y es, pues, en la lucha antiimperialista, y en nombre de ella, como era preciso construir desde los obreros, los campesinos, las capas medias y un sector de la burguesía nacional, una oposición común a la dominación de la oligarquía y del capital extranjero. Esta alianza interclasista era la base de la construcción de lo popular-nacional. Martuccelli subraya que su exclusión de toda gestión gubernamental, por un lado, deslegitimó a un sistema político ya de por sí débilmente representativo, y, por el otro lado, impidió darle un rostro político consistente al Pueblo. Asimismo, la espeficidad de la experiencia chola y popular limeña fue ajena a la comprensión del APRA.

El gobierno militar, por su parte, mediante una ideología nacional-desarrollista (proporcionado por el CAEM) y un espíritu común, poseía una visión amplia de la seguridad nacional. El gobierno

2 Para el autor, el pueblo se convirtió en el gran actor político del siglo XX, ya sea como pueblo-clase, puebloraza o pueblo-nación. El pueblo designará así a los sectores "olvidado" y "marginados", a "los de abajo" y al Perú "profundo", con el propósito de recrear nación, pero tendrá dificultades para su realización. 
de Velasco puso en la práctica muchas de las medidas propugnadas por el APRA desde los años treinta: nacionalización de empresas extranjeras, participación de los trabajadores en la propiedad y reforma agraria. Buscó el desarrollo hacia adentro a través de la puesta en marcha de reformas que permitieran eliminar la "dependencia externa"y la "dominación interna", causas de la "desunión nacional"y del "desencuentro" entre el pueblo y las Fuerzas Armadas.

¿Y Cuáles fueron las limitaciones del gobierno militar a la hora de construir lo popularnacional?

En principio, la desconfianza de los militares hacia la sociedad civil y su disidencia a formar un movimiento-partido de masas que pueda asegurar la movilización y el encuadramiento que necesitaba. Si bien, esta limitación reflejaba la estructura jerárquica y autoritaria del gobierno militar, también reproducía la división clasista y étnica de la sociedad peruana, y la desconfianza que las élites proyectaban hacia los sectores subalternos. No obstante, a pesar de esta limitación en la construcción "desde arriba" de lo nacional popular, los años de la década de 1970 fueron testigos de una estructura política de la sociedad civil, y lo más importante: la construcción de lo popular-nacional adquirió autonomía política, proceso que fue el resultado de órdenes de diferente realidad, a saber: (1) el éxito del gobierno militar en la construcción de lo popular se dio en el ámbito de la cultura. Sus políticas culturales y educativas transformaron en profundidad el imaginario nacional; y (2) el cambio radical de la composición interna de los sectores populares en esos años, como fenómeno migratorio.

No obstante, existen tres procesos en los que lo popular irrumpe en Lima: éxitos electorales de la izquierda peruana que, entre 1984-1987, con Alfonso Barrantes gana la Municipalidad de Lima. Segundo, la aparición de Sendero Luminoso y de la lucha armada. Tercero: la conciencia política sobre los ambulantes. Martuccelli señala que nos es gratuito que la década del ochenta sea uno de los grandes momentos de creatividad de las ciencias sociales en el país.

Para García, en el fondo, la renovación de la alianza política con los sectores populares dependía más del poder persuasivo del líder, que del despliegue eficaz del aparato partidario, no poseía ninguna representación idónea de lo popular-nacional y tampoco tenía ningún proyecto explícito al respecto debido a su mirada economicista.

Después del intento fallido del primer gobierno de García, del desmoronamiento electoral y político de la izquierda, y del fin de Sendero Luminoso, el Perú no ha sido más el teatro de ningún verdadero proyecto de construcción política del pueblo. El pueblo, como imaginario y como práctica política, perdió consistencia y protagonismo, ya no constituye parte de la retórica ni objetivo de las movilizaciones sociales. Los gobiernos de Fujimori, Toledo, el segundo gobierno de García y Humala, le han dado diferentes connotaciones, pero ninguno logró articular una auténtica política en torno a lo popular. Desde los ochenta, como resultado de la crisis del crecimiento económico y la descomposición de la sociedad, el pueblo deja de ser representado únicamente como un actor colectivo y se convierte en un conjunto de experiencias sociales y populares que se individualiza en sus expectativas; cambio que dio paso al inicio del gobierno chicha, con Fujimori, así como al surgimiento del outsider. 
El segundo capítulo analiza el vigor de las fronteras y jerarquías culturales pero también de sus primeros desplazamientos y puestas en cuestión. Parte de la idea que la crisis política de la noción del pueblo, no fue sino una de las fuentes del cambio estructural que ha conocido Lima. Para Martuccelli, más allá de los términos, la cuestión central que subyace a estos procesos, no es otra cosa que la transformación del imaginario nacional. Lima, en las últimas décadas, ha terminado por engendrar una cultura propia, profundamente peruana, pero que no representa, en su totalidad, al Perú de hoy.

Este proceso de conflicto cultural estaría marcado por la presencia de cuatro grandes jerarquías culturales: lo criollo, lo huachafo, lo cholo y el racismo. Cada una de ellas tiene sus propias características, pero es a partir de estos procesos que se construyó el perímetro de la arena conflictiva socio-cultural limeña.

Para Martuccelli, lo criollo constituye la primera gran identidad cultural de Lima, y su centro está en su distinción y distancia de lo andino. En el siglo XX, el término ${ }^{3}$ no dejó de resignificarse, pero conservó un elemento central: el criollo era el que no era serrano, era sobre todo el que no era recién llegado de la sierra. Pero más allá de esta caracterización general, lo criollo está lejos de ser evidente. Lo criollo se definió más en referencia y distancia a la cultura andina que en referencia y contraste con las formas culturas extranjeras. Lo esencial de esta invención de lo criollo no fue una "resistencia" a las influencias externas, sino la voluntad de diferenciarse de lo andino. Y precisamente la migración andina habría constituido un factor central en la pérdida de protagonismo y la hegemonía de la cultura criolla, y con ello la imagen de una Lima colonial.

Lo huachafo es visto como una estrategia de distinción social y cultural criolla. El uso del término se remonta a los inicios del siglo XX, en la Lima de los años 1940-1960, el designar a alguien como huachafo era un acto evidente de distinción cultural y de posicionamiento clasista, activado sobre todo por la puesta en peligro de su exclusividad en el consumo de los bienes y servicios por la llegada de nuevos consumidores y, por lo general, estigmatizados por su mal gusto. Lo huachafo, centralmente, es definido por la distinción social y cultural desde el consumo. Las clases medias en Lima se organizaban, entonces, alrededor de una doble frontera: la decencia y la separación entre aquellos que manifestaban buen gusto de todos aquellos que no gozaban de él. Lo huachafo también podía, por momentos, designar una acción, y no necesariamente, un estado social. Todo el mundo podía en un momento $u$ otro ser tildado de huachafo. Fue una categoría de descalificación cultural en la Lima que se imaginaba señorial.

Por otro lado, lo cholo está asociado con la migración que experimentó la ciudad desde 1950, y sobre todo en la década de 1960. Martuccelli describe los cambios demográficos que este fenómeno generó y que globalmente se trató de un periodo donde la visibilidad urbana del fenómeno, contrastó con la invisibilidad del cambio social y cultural en curso. A partir de una revisión de distintos planteamientos, considera que lo cholo supuso el gran descentramiento del país en términos políticos y demográficos: de una sociedad terrateniente y de población rural, a un país urbano y de mestizaje urbano-cultural. Sin

3 Para el autor, el término aparece recién a mediados del siglo XIX, es solamente en la primera mitad del siglo XX que el término y la noción de cultura criolla se consolidan como diferencia de la cultura limeña de expresiones culturales que venían de otras regiones del país. Es decir, como toda tradición, también lo criollo fue inventado, y sobre todo asociado a la música. 
embargo, los migrantes no se aculturan, sino que van paulatinamente a engendrar una cultura popular que, progresivamente, y por vías inesperadas, se volverá mayoritaria. En Lima, ni la cultura andina o limeña triunfó una sobre la otra. Pero, según Martuccelli, el fenómeno cholo, aporía étnica y social, no desarrolló una genuina cultura chola, no dio origen a una cultura propia: no existe una cultura chola.

El tercer capítulo, de manera secuencial, aborda las dimensiones propiamente urbanas y económicas de las transformaciones vividas en Lima y, por lo general, para el autor, se condensan en el llamado sector informal. Lima es definida como una ciudad sin rostro y, a partir de las ideas de Francisco Durand, es representada como una sociedad fracturada, donde lo legal, lo informal y lo delictivo confluyen en la práctica de los actores. Pero esta fractura también se expresa en la definición de los lugares de la ciudad en función de orientaciones geográficas: Lima Tradicional, Lima Sur, Lima Norte y Lima Este, que hace inteligible la realidad de una ciudad en la que sus habitantes se quedaron sin mapa cognitivo. Los individuos desconocen las localidades de su ciudad.

El cruce entre la migración y el mercado del trabajo le permiten interpretar la informalidad en la ciudad limeña. En Lima, para el autor, al menos en su origen, la informalidad testimonia tanto el fracaso del sector privado y del Estado. Sin embargo, su expansión es indisociable de la profundidad de la crisis económica de la década de los ochenta. La migración del campo a la ciudad no solo cambió la cuestión cultural y urbana de la ciudad, también transformó la economía de Lima.

La principal legitimidad, según el autor, no vino del ni fue al mercado, sino que se organizó en torno al consumo. En el imaginario de los actores prima el consumo. Considera que hablar de ciudadanía desde el consumo es un exceso, pero rescata las reflexiones e interrogantes sobre la participación social a partir del acceso a bienes públicos de consumo. Así, el principal impacto del sector informal a nivel de consumo, en los noventa, se dio a nivel de los bienes públicos.

Un punto de reflexión importante aquí es sobre el sentido de la casa propia y su rol en la estimulación del individualismo. La casa propia sirvió como sueño y soporte, incluso como concresión del mito del progreso. La casa propia tiene un origen en un proceso colectivo. La casa propia no fue la expresión de la invasión andina de la ciudad que algunos vaticinaron, sino más bien fue un elemento decisivo de la afirmación individualista de los sectores populares, de sus diferenciaciones internas, e incluso, de la seducción que sobre algunos de ellos ejercieron los principios de la economía de mercado. Este último coincide con la tesis que formuló Hernando de Soto, quien al mismo tiempo le dio forma ideológica. Respecto a la relación entre el individualismo y la casa propia, precisa:

"Pero en Lima, la casa propia no fue un efecto directo del individualismo de los sectores populares. Lo contrario es más cierto: fue la casa propia lo que estimuló progresivamente su individualismo" (p. 127).

Martuccelli no presta ninguna atención a la posesión del espacio con fines mercantiles y para el enriquecimiento, en especial sobre el tráfico de terrenos ${ }^{4}$, fenómeno que se

4 El tema ha sido desarrollado en una investigación etnográfica reciente. Véase Pimentel Sánchez, Nekson (2017). Subalternos autónomos. Transformaciones periurbanas y tráfico de tierras en el contexto de una 
sobrepone a la ocupación por necesidad, sea esta de manera individual o colectiva. Sin duda, esto se debe a la ausencia de fuentes respecto a esta problemática que es tan actual pero no por ello de origen reciente, y donde interactúan múltiples actores que se circunscriben dentro de la legalidad, esto es, la instrumentación de herramientas legales y organizaciones colectivas para cubrir actos ilícitos.

Hasta aquí la primera parte. La segunda parte, y tomando en cuenta los procesos hasta aquí analizados, el ensayo propone una interpretación de la realidad limeña actual, y para ello plantea el análisis de tres grandes arenas. En primer lugar, la novedad y la especificidad de la modernización limeña actual, el proyecto reglamentador. En segundo lugar, los procesos y las formas por las cuales una sociabilidad común se ha creado e impuesto en la ciudad. Y por último, los perfiles propios del individualismo metonímico limeño.

El cuarto capítulo analiza el proyecto reglamentador, el elemento más significativo del proyecto de modernización que experimentó Lima en la década del noventa y que, desde entonces, se desarrollaría a través de una dinámica que va mucho más allá de la sola cuestión económica entre formalización e informalidad, y que se estructura a través de un conjunto de distintas políticas y medidas que buscan impulsar, no sin contradicciones, la consecución del orden a través de las reglas. Lo central de este proyecto estaría en la voluntad de una puesta en regla reciente de las relaciones sociales. El proyecto reglamentador sería mucho más importante que el neoliberalismo en el Perú.

Señala dos aspectos del neoliberalismo en el Perú. El neoliberalismo no fue en lo esencial un proyecto conservador. $Y$ en segundo lugar, el neoliberalismo peruano fue una propuesta que se legitimó en sus inicios en nombre del orden, pero que, pasada la primera fase de shock y de implementación, lo hizo, sobre todo, en nombre de una mayor eficiencia macroeconómica y administrativa. Y que no fue, en absoluto, un proyecto ideológico que apuntó a la producción de un individuo neoliberal.

Aquí Martuccelli olvida las maquinarias de los medios de comunicación que reproducen pautas e ideas de políticas neoliberales, así como las resistencias que existen por parte de los grupos económicos de poder que tienen presencia en el Estado a partir de redes clientelares, patronazgo y lobbismo. Además, toda idea para que tome cuerpo y sentido tiene que ser adoptada por la sociedad, por lo que podemos decir que los individuos han reproducido los principios del neoliberalismo en sus prácticas cotidianas.

La informalidad (económica y normativa) es tomada como un elemento a partir del cual se ponen en forma y se representan las relaciones sociales. Es decir, la informalidad, a diferencia de la ilegalidad, se habría convertido en el imaginario del lazo social de la ciudad. La ilegalidad no sería, a diferencia de otros países de la región, la matriz de las relaciones sociales e interpersonales. Lo informal es representado como el gran rostro de la transgresión. Precisa que la reglamentación creciente de la cotidianidad es uno de los grandes cambios de la sociedad limeña.

Sin embargo, este proceso de reglamentación tendría límites, sobre todo, debido a que no siempre se traduce en un retroceso de la informalidad y de la ilegalidad. Pero a pesar de estas limitaciones, busca afirmarse a través de un conjunto de espirales caleidoscópicas

sociedad de mercado: Carabayllo, Lima. Tesis. Lima: UNMSM. Pero también existen algunas reflexiones en la última edición La ciudad llegal de Julio Calderón (2016). 
que se inscriben dentro del imaginario de la matriz informal de las relaciones sociales.

El quinto capítulo analiza la revolución de la sociabilidad en la ciudad limeña actual. A pesar de la transformación urbana y de la diversidad demográfica, bajo el fondo de desigualdad social y económica, Lima estaría enmarcada por una sociabilidad urbana común. Y, tras ella, por la percepción de vivir en una misma ciudad.

¿Cuáles son los grandes rasgos de esta sociabilidad? ¿Y cuál es su relación y diferencia con la hegemonía? Son preguntas centrales que articulan este acápite. Para el autor, la sociabilidad no está asociada al triunfo de una contra-hegemonía popular. Si bien considera importante la contribución de Gramsci, considera que no es pertinente para la interpretación de la sociedad limeña actual. Para el autor, Lima experimenta, por un lado, la caducidad de la impronta cultural hegemónica de las clases dirigentes, y por el otro, la formación, no de una contra-hegemonía popular, sino, de una sociabilidad común. Enfatiza que Lima es "una ciudad altamente fragmentada, marcada, no obstante, por una asombrosa sociabilidad común. Los limeños comparten un estilo común, por desprolijo que muchos lo juzguen" (p. 196).

La sociabilidad limeña ha conocido transformaciones que, marcadas por otros procesos estructurales, habría seguido un curso independiente de ellos. La sociabilidad es un estilo de relación interpersonal que ha transformado de forma masiva a la sociedad limeña y en todos sus grupos sociales.

¿Cuál es el proceso de formación y de expansión de esta sociabilidad común? Para la comprensión de la sociabilidad limeña se debe tomar en cuenta la fusión, término proveniente de la música, antes que el mestizaje, ya que esta última lleva a resultados opuestos a la que conduce el tema de la fusión. La fusión permite pensar la efervescencia limeña actual no desde la raza, ni desde la ambigüedad de la cholificación o su triunfo simbólico, sino a partir de manifestaciones propiamente culturales y sociales. El mestizaje, detrás del monolitismo de la ideología de la mezcla, habría estado al servicio de la separación categorial de las personas; mientras que la fusión, detrás de la heterogeneidad de sus prácticas, al servicio de la desinserción categorial de los individuos. La fusión permitiría comprender mejor la especificidad de la sociabilidad limeña y lo que realmente selló el cambio.

En la asociación de la fusión con la música chicha, esta sería de la especificidad de la experiencia de los migrantes en la ciudad de Lima, debido a que la música chicha no está, como la ideología mestiza, al servicio del imaginario del crisol de razas; a través de la fusión de aires andinos con ritmos tropicales, antes de abrirse a otras sonoridades, la música chicha expresó una trayectoria social distinta: el quiebre y la reelaboración del mundo andino en la ciudad en medio del colapso de las formas tradicionales limeñas. La música chicha fue, sobre todo, un acto de sucesión cultural con la matriz andina y una de las vías por las cuales transitó el imaginario de la fusión, y tras él, la aparición de una sociabilidad. Hasta que la fusión no se convertía en el corazón del nuevo imaginario limeño, era posible oponer lo culto, lo formal, lo limpio a lo inculto, lo informal, lo sucio y lo popular. El valorfusión transformó estas oposiciones. Y la chicha, para el autor, trata de una verdadera coproducción ciudadana. Precisa que la penetración de la cultura chicha en el ámbito político-institucional fue a través de la pareja Fujimori-Montesinos. 
Otro gran elemento de la sociabilidad limeña contemporánea es el humor del aplaste, donde prima la puesta en comicidad la propia victoria y la humillación del otro. El humor del aplaste comporta una guerra de todos contra todos: los jóvenes contra los viejos, las mujeres contra los hombres, los ricos contra los pobres, los limeños entre sí y contra los provincianos, y en todos lo que está en el centro es el aplaste.

En este escenario, el achorado aparece como la expresión extrema de la cultura chichay del humor del aplaste. El achorado avasalla sin miramientos, por ignorancia, por indiferencia, por buscavidas. El achorado, debido a la ausencia del respeto de las instituciones y a la existencia de una sociedad desigual, busca hacerse respetar individualmente, avasalla a los demás y a las instituciones. No busca competir sino salvarse. Pero para Martuccelli, el achorado es empero también la expresión ambivalente de la fuerza de la democratización que vive la sociedad limeña, una democratización que no ha tenido su epicentro ni en la economía, ni en la política y ni tan siquiera en la cultura, sino la sociabilidad. El achorado es una figura de contestación de jerarquías. Pero, a pesar de estas tendencias de una supuesta democratización, la sociedad limeña estaría aún marcada y envuelta por consideraciones raciales, con usuarios en todas las categorías sociales. Además, en Lima, la horizontalidad interactiva estaría disociada de la igualdad ciudadana, así como expresa la siguiente cita:

"(...). Lo que produjo la revolución de la sociabilidad es otra cosa: una puesta en jaque universal, pero solo interactiva, de las jerarquías. Lo que la revolución de la sociabilidad limeña señala en sus distintos elementos, todos ellos, en la misma dirección pero no al unísono, es la emergencia de nuevas reglas en un estricto ámbito relacional. En este sentido, Lima es menos, mucho menos, el teatro de una expansión de la igualdad que de una arena que lidia y puesta en jaque continua de las verticalidades interactivas" (p. 242).

El sexto, y último capítulo, analiza los perfiles del individualismo limeño desde la experiencia social e histórica de la ciudad. Para el autor, Lima no es más una sociedad colonial o estamental, sino una ciudad de individuos, cuya modernización en las últimas décadas del siglo XX estuvo marcada por una intermediación cultural antes que por una intermediación política.

El individualismo limeño debe entenderse como una variante del individualismo agentico latinoamericano.

"Se trata de un individualismo que encuentra, a nivel del individuo y de sus habilidades, en mucho representadas como ajenas o paralelas a las instituciones y a sus prescripciones, su foco central. (...)" (p. 246).

El corazón del individualismo limeño, para Martuccelli, es más social y cultural que estatal o político. Lima constituye individuos que no son esencialmente el resultado de prescripciones institucionales, sino, casi al contrario, un individuo que se percibe como un individuo híper-actor.

El individualismo metonímico existe en el marco de un conjunto dentro del cual se posiciona. El actor metonímico es incomprensible fuera de su referencia a esta globalidad. Sin embargo, esta referencialidad es percibida como externa, en realidad, está marcada y delimitada por las miradas de algunos otros de las que es particularmente sensible. Es en este sentido que el actor es más metonímico que egoísta. La regla se modula, los actores 
acatan más la ley del grupo al cual pertenecen que la ley abstracta de la sociedad como grupo de referencia.

En primer lugar, esta comprensión generalizada a la desviación distingue claramente entre la viveza y la transgresión propiamente dicha de las reglas. En segundo lugar, los limeños, en el fondo, y a pesar de la creciente puesta en regla de la vida cotidiana por el proyecto reglamentador, muchas veces tienden a tener más confianza en los individuos que en las reglas. Los actores tienen muchas veces más confianza en los individuos que en la mayoría de las normas o de las instituciones. En ese sentido, y a pesar de la expansión del proyecto reglamentador, la aplicación ecuánime y universal de la ley no es nunca, entera y cabalmente, un ideal colectivo.

Los rostros del individualismo limeño son el criollo, el achorado, el propietario. Cada uno de ellos vive de la oportunidad. Pero ahí donde el criollo tiene que saber aprovechar la oportunidad que se presenta y cuando se presenta, y el achorado se rebela contra la falta y la desigualdad de las oportunidades, el migrante-propietario crea, pacientemente, la oportunidad. En los tres casos, está empero en juego una habilidad, contrariamente a lo que muchas veces se indica, profundamente optimista y furiosamente individualista. La oportunidad criolla, el atropello achorado y la acumulación predial son tres caras de una misma realidad social: la de individuos que sienten que tienen que lidiar, con ingenio, con fuerza o con esfuerzo, en medio de las arenas urbanas.

El individualismo metonímico tiene dos dimensiones: "heroica" e individuo-relacional. Los soportes del individuo no se encuentran pues principalmente en las instituciones, los derechos o en el trabajo asalariado, sino que tienen que ser producidos -o al menos sostenidos o recreados- por los propios individuos. Es desde las capacidades personales y soportes no institucionales -sobre todo familiares- desde donde organiza su metonimia. Frente a los embates de la vida, y ante las insuficiencias de los servicios o de los derechos, la familia es el principal y a veces el único soporte, real o imaginario. Lo que constituye, sin duda, un importante desafío: el proyecto reglamentador busca aplicarse en medio de una ciudad sumida en una profunda desconfianza institucional.

En Lima, se produce un individuo que tiene conciencia de hacerse cargo de sí mismo y de algunos otros, con algunos otros, en medio de una serie de arenas en las que siempre tiene que estar en vigilia y al acecho. Lima, así, es una "arena polimorfa de luchas".

$Y$ finalmente, a modo de conclusión, hace inteligible seis tesis importantes: (1) respetando la pluralidad y la contingencia de los procesos, es posible proponer una interpretación social e histórica global de la transformación. (2) El gran cambio en Lima se explica menos desde la crisis o las necesidades estructurales que desde las respuestas imaginativas que a cada uno de estos desafíos dieron los actores. (3) El gran cambio alteró en profundidad las relaciones sociales entre los individuos sin modificar las relaciones estructurales de poder. (4) En Lima, la informalidad designa, más allá de economía, una matriz normativa y estilística del lazo social contra la cual se yerguen diversos procesos de puesta en regla de la vida social. (5) Las ciencias sociales peruanas, a través de muchas veces de una paradójica adhesión a la lógica del peso de las herencias, no han cesado de comprender la novedad limeña. (6) El Perú, marcado por la centralización capitalina en lo 
que concierne a las relaciones de similitud y de diferenciación entre Lima y el país, estaría un periodo inédito de construcción entre Lima y las regiones de la nación peruana.

Los pensamientos de Martuccelli no son ajenos a la formación de una nación y de una ciudadanía política que pueda encontrar-desde Lima- un asidero identitario común, pero implicaría que el Perú, y no solamente Lima, desde su innegable y genuina especificidad, se reconozca como una nación de ciudadanos. Y sin duda, las líneas de este ensayo constituyen una buena puerta de entrada para el debate y la investigación sobre los aspectos de la ciudad que producimos y vivimos, pero que, al mismo tiempo, aún nos molesta. 